\title{
Photodarkening as a heat source in ytterbium doped fiber amplifiers
}

\author{
Peter Šušnjar, Vid Agrež, ${ }^{*}$ And Rok PetKovšek \\ University of Ljubljana, Faculty of Mechanical Engineering, Aškerčeva 6, SI-1000 Ljubljana, Slovenia \\ vid.agrez@fs.uni-lj.si
}

\begin{abstract}
Theoretical and experimental evaluation of the photodarkening effect as a heat source in ytterbium doped fibers is presented. An additional non-radiative decay channel that opens after photodarkening the fiber is identified via fluorescence lifetime reduction and as an additional heat source proportional to inversion. It is included in the heat source model which was tested on a core-pumped fiber amplifiers. High temperature elevation at low pump powers shows potential heat-related problems in high inversion systems that are more susceptible to photodarkening.

(C) 2018 Optical Society of America under the terms of the OSA Open Access Publishing Agreement

OCIS codes: (140.3510) Lasers, fiber; (140.3615) Lasers, ytterbium; (060.2270) Fiber characterization; (140.6810) Thermal effects.
\end{abstract}

\section{References and links}

1. D. C. Brown and H. J. Hoffman, "Thermal, stress, and thermo-optic effects in high average power double-clad silica fiber lasers," IEEE J. Quantum Electron. 37(2), 207-217 (2001).

2. J. W. Dawson, M. J. Messerly, R. J. Beach, M. Y. Shverdin, E. A. Stappaerts, A. K. Sridharan, P. H. Pax, J. E. Heebner, C. W. Siders, and C. P. Barty, "Analysis of the scalability of diffraction-limited fiber lasers and amplifiers to high average power," Opt. Express 16(17), 13240-13266 (2008).

3. C. Jauregui, H.-J. Otto, F. Stutzki, J. Limpert, and A. Tünnermann, "Simplified modelling the mode instability threshold of high power fiber amplifiers in the presence of photodarkening," Opt. Express 23(16), 20203-20218 (2015).

4. R. Peretti, C. Gonnet, and A.-M. Jurdyc, "Revisiting literature observations on photodarkening in Yb3+ doped fiber considering the possible presence of Tm impurities," J. Appl. Phys. 112(9), 093511 (2012).

5. J. J. Montiel i Ponsoda, M. J. Söderlund, J. P. Koplow, J. J. Koponen, and S. Honkanen, "Photodarkeninginduced increase of fiber temperature," Appl. Opt. 49(22), 4139-4143 (2010).

6. S. Jetschke, S. Unger, A. Schwuchow, M. Leich, and M. Jäger, "Role of Ce in $\mathrm{Yb} / \mathrm{Al}$ laser fibers: prevention of photodarkening and thermal effects," Opt. Express 24(12), 13009-13022 (2016).

7. H.-J. Otto, N. Modsching, C. Jauregui, J. Limpert, and A. Tünnermann, "Impact of photodarkening on the mode instability threshold,” Opt. Express 23(12), 15265-15277 (2015).

8. C. Jauregui, H.-J. Otto, S. Breitkopf, J. Limpert, and A. Tünnermann, "Optimizing high-power Yb-doped fiber amplifier systems in the presence of transverse mode instabilities," Opt. Express 24(8), 7879-7892 (2016).

9. S. Jetschke, S. Unger, U. Röpke, and J. Kirchhof, "Photodarkening in Yb doped fibers: experimental evidence of equilibrium states depending on the pump power," Opt. Express 15(22), 14838-14843 (2007).

10. R. Piccoli, D. Mechin, T. Robin, and S. Taccheo, "Lifetime reduction due to photodarkening phenomenon in ytterbium-doped fibers and rate equation term," Opt. Lett. 38(21), 4370-4373 (2013).

11. S. Jetschke, A. Schwuchow, S. Unger, M. Leich, M. Jäger, and J. Kirchhof, "Deactivation of Yb ${ }^{3+}$ ions due to photodarkening," Opt. Mater. Express 3(4), 452-458 (2013).

12. M. Engholm and L. Norin, "Preventing photodarkening in ytterbium-doped high power fiber lasers; correlation to the UV-transparency of the core glass," Opt. Express 16(2), 1260-1268 (2008).

13. R. Paschotta, J. Nilsson, A. C. Tropper, and D. C. Hanna, "Ytterbium-doped fiber amplifiers," IEEE J. Quantum Electron. 33(7), 1049-1056 (1997).

14. S. Jetschke, S. Unger, M. Leich, and J. Kirchhof, "Photodarkening kinetics as a function of Yb concentration and the role of Al codoping," Appl. Opt. 51(32), 7758-7764 (2012).

\section{Introduction}

The advance of active fiber technology allows for reaching high output powers from fiber lasers. Till now the focus of the research was on lowering the damage threshold while retaining the high quality of the beam output. Other thermal effects were neglected firstly due to good cooling capabilities of the fiber geometry and secondly by selecting ytterbium doped fibers allowing for low quantum defect which is believed to be the main heating source. 
Based on Brown's early study [1] on thermal and thermo-optic effects in high-power silica fiber lasers the limit was set to $36 \mathrm{~kW}$ of diffraction limited power extractable from a single fiber laser which is imposed by thermal (thermal lensing) and stimulated Raman scattering effects [2]. However, thermally induced changes of refractive index lead to instabilities in transverse beam profiles at much lower output powers of few $\mathrm{kWs}$ [3]. It is important to note that when deriving this upper limit, quantum defect was considered as the only (relevant) heating mechanism.

However, ytterbium doped fibers are susceptible to light induced losses due to photodarkening (PD). This loss grows by time, but saturates at certain point. The time and the level at which this occurs depend on fiber manufacturing process especially on ion composition, density of excited ions and temperature [4]. Beside the reduced efficiency of ytterbium based fiber laser systems, the problematic aspect of the effect is also the additional heat load due to absorption of pump and signal light in PD-induced color centers [5]. While it was shown that cerium co-doping can significantly reduce the amount of $\mathrm{PD}$, an additional heat source was established by interaction of cerium and ytterbium ions [6]. Also the PD effect was linked with transverse mode instabilities (TMI) encountered in high power photonics crystal fiber lasers. Despite almost negligible impact on laser efficiency due to PD induced loss, TMI threshold fell by half [7]. To address this, PD was included as additional heat source in TMI models represented as linear absorption term [3]. In general, minimizing PD by core material composition is advised as the solution for increasing the output power of high-power fiber lasers limited by TMI. When not possible, active ion concentration and the core conformation should be optimized to reduce TMI [8].

As the PD equilibrium excess loss almost linearly increases with excited ion density [9] it can represent the mayor heat source in the core pumped fiber preamplifiers used for ultrashort pulse amplification. There, short fiber lengths are required to minimize nonlinear effects and therefore highly doped fibers are used for which PD is more intense. PD is further enhanced in regions with saturated inversion due to low signal power.

In this work, a contribution of different effects to heating of the ytterbium doped fiber preamplifier is evaluated with the emphasis on the comparison between quantum defect and PD. For this purpose, a theoretical model is presented and compared to the measured data showing good agreement. Further, the lifetime reduction of the photodarkened fiber $[10,11]$ due to the non-radiative decay is investigated. The reduction was included in the model and extracted value agrees with measured lifetimes. The findings are then tested on the experimental setup of a typical core pumped preamplifier where again a PD effect is found to be the dominant heat source in the first region where it can cause the polymer or splice failure if not addressed correctly.

\section{Heat load model}

A temperature rise $\Delta \mathrm{T}$ for a core-pumped optical fiber is given by [1]

$$
\Delta T=T_{\text {surf }}-T_{\infty}=\frac{Q a^{2}}{2 b h}
$$

where $a$ and $b$ are core and a clad radius respectively $T_{\text {surf }}$ and $T_{\infty}$ are temperature at the fiber surface and of the surroundings respectively, $Q$ is a volumetric heat source and $h$ is a heat transfer coefficient for free convection. Here, heat flow along fiber axis has been neglected as the temperature gradient in this direction is sufficiently small in most of the cases except at the splices of passive and active fiber, where heat source suddenly drops to zero.

The total heat source $Q$ (per volume unit) is a sum of different contributions $Q=Q_{\mathrm{ABS}, 0}+$ $Q_{\mathrm{QD}}+Q_{\mathrm{PD}}$. Here, $Q_{\mathrm{ABS}, 0}$ is the volumetric heat source due to the initial intrinsic loss of pump and signal light, i.e. initial linear absorption of a pristine fiber as it was observed by experiment 


$$
Q_{A B S, 0}=\alpha_{0, s} \Gamma_{s} P_{s} / A+\alpha_{0, \mathrm{p}} \Gamma_{p} P_{p} / A .
$$

Next, $Q_{\mathrm{QD}}$ is a heat source due to quantum defect, i.e. an energy difference between pump and signal photon $\Delta E_{\mathrm{s}}$ or spontaneously emitted one $\Delta E_{\mathrm{SE}}$ (mean energy difference determined from fluorescence spectrum)

$$
Q_{Q D}=\left(W_{21} \Delta E_{s}+A_{21} \Delta E_{S E}\right) n_{2} N_{t o t} .
$$

Finally, $Q_{\mathrm{PD}}$, a heating source related to heating due to $\mathrm{PD}$ is

$$
Q_{P D}=Q_{L A}+Q_{N R}=\alpha_{\mathrm{PD}, s} \Gamma_{s} P_{s} / A+\alpha_{\mathrm{PD}, \mathrm{p}} \Gamma_{p} P_{p} / A+A_{N R} n_{2}\left(P_{p}, P_{s}\right) N_{t o t} \Delta E_{0} .
$$

As proposed by [11], there are two distinguishable loss mechanisms related to PD, i.e. linear absorption of pump and signal light in PD induced color centers $\left(Q_{\mathrm{LA}}\right)$ and nonradiative relaxation of excited ytterbium ions $\left(Q_{\mathrm{NR}}\right)$. The first one can be observed as modification of absorption spectrum of ytterbium doped fiber with emerging broad peak around $500 \mathrm{~nm}$ and extending all the way to laser transitions of ytterbium ions around 1000 $\mathrm{nm}$ [12], while the second one was measured as a shortening of a fluorescence lifetime $[10,11]$. Other quantities in upper definitions are an intrinsic loss absorption coefficients $\alpha_{0, \mathrm{p} / \mathrm{s}}$ at pump and signal wavelength, an overlap integral $\Gamma$, a core area $A$, stimulated emission, spontaneous emission and non-radiative decay rates $W_{21}, A_{21}$ and $A_{\mathrm{NR}}$, population inversion $n_{2}$, volumetric density of ytterbium ions in the fiber core $N_{\text {tot }}$, absorption coefficient of PD induced color centers $\alpha_{\mathrm{PD}, \mathrm{p} / \mathrm{s}}$ and an energy difference between laser levels $\Delta E_{0}$.

Combining Eqs. (1)-(4) we derive the relation for temperature rise in the dependence on experimental parameters i.e. fiber geometry, doping, cross-sections, pump and signal powers $\left(P_{\mathrm{p}}\right.$ and $\left.P_{\mathrm{s}}\right)$ etc.

$$
\Delta T=\frac{a^{2}}{2 b h}\left[\frac{\Gamma}{A}\left(\alpha_{p} P_{p}+\alpha_{s} P_{s}\right)+W_{21} \Delta E_{s} n_{2} N_{t o t}+\left(A_{21} \Delta E_{S E}+A_{N R} \Delta E_{0}\right) n_{2} N_{t o t}\right] .
$$

Here, $\alpha_{\mathrm{p} / \mathrm{s}}$ are net pump and signal absorption coefficients as a sum of intrinsic and PD related linear absorption. The first part in (6) linearly depends on pump and signal power and grows in time until PD process saturates. The second is the major part of quantum defect contribution, which also increases with signal power as well as inversion level, while the last one doesn't scale with power and is therefore not critical when scaling power of fiber lasers. However, we included it in the model to relate the observed changes in fluorescent lifetime with this additional, though very weak, heat source.

\section{Experimental}

In all experiments a core pumped $10 / 125 \mu \mathrm{m}$ fiber with ytterbium ion density of $4.3 \times 10^{25}$ $\mathrm{m}^{-3}$ was used. The PD induced loss in a $10 \mathrm{~mm}$ long fiber core pumped by $976 \mathrm{~nm}$ light was measured using a $633 \mathrm{~nm}$ probe at different power levels $(>70 \mathrm{~mW})$ at which inversion population saturates.

Our heat source model was tested on a $2 \mathrm{~mm}$ short piece of an active fiber to avoid effects due to amplified spontaneous emission and reabsorption as well as to keep the inversion homogeneous within the whole piece which allowed for more direct comparison between the model and experiment. A pump diode at $976 \mathrm{~nm}$ and a signal diode at $1030 \mathrm{~nm}$ (both single mode) of varying power were used for excitation. The pump diode rise time was less than 20 $\mu \mathrm{s}$. The ratios of absorption coefficients at signal and pump wavelength $\alpha_{\mathrm{p}} / \alpha_{\mathrm{s}}=0.105$ and 0.227 were experimentally determined by comparing a temperature increase at same signal and pump powers for pristine and photodarkened fiber, respectively. Temperature rise was measured as a difference of the temperature of the active and passive part of the fiber in thermally stable environment using thermal camera with a close-up objective. Temperature was stable within $0.2^{\circ} \mathrm{C}$. Assuming a free (natural) convection as the only heat dissipation mechanism, a heat transfer coefficient $h=150 \mathrm{~W} /\left(\mathrm{m}^{2} \mathrm{~K}\right)$ was calculated for a horizontal 
cylinder. Fluorescent light was collected by a $105 / 125(\mathrm{NA}=0.22)$ multimode fiber placed perpendicular to the active fiber and guided to a spectrometer or a photodiode to measure spectrum of spontaneously emitted light or fluorescence lifetime respectively. From the average wavelength of spontaneously emitted light $\Delta E_{\mathrm{SE}}=45 \mathrm{meV}$ was determined, which is slightly lower than quantum defect related to difference in energy of signal and pump photon $\Delta E_{\mathrm{SE}}=68 \mathrm{meV}$. For fluorescence lifetime measurement, the fiber was excited by $5 \mathrm{~ms}$ long pulses with $50 \mathrm{~Hz}$ repetition.

The same monitoring setup used for model testing on a short piece of fiber was then applied to a core-pumped preamplifier based on the same active fiber with the length of 38 $\mathrm{cm}$. Fluorescence and temperature were measured along the fiber length.

In all cases the measurements were first performed on a pristine fiber with short illumination times to avoid significant PD of the examined fiber during the measurement. Next, fiber was exposed to $976 \mathrm{~nm}$ light for long enough to reach loss saturation level according to previously conducted tests with $633 \mathrm{~nm}$ probe light. Finally, the same set of measurements was repeated with a photodarkened fiber.

\section{Results and discussion}

The presented heat-load model was first evaluated on a 2-mm long piece of active fiber at different power levels of signal diode $(0,100$ and $200 \mathrm{~mW})$. The results are shown in Fig. 1. The population of upper laser level as determined from the power of spontaneously emitted light, was compared to results of a simple 2-level model [13] (Fig. 1(a)). The measured values fit to the model within measurement uncertainty for all three signal light powers.
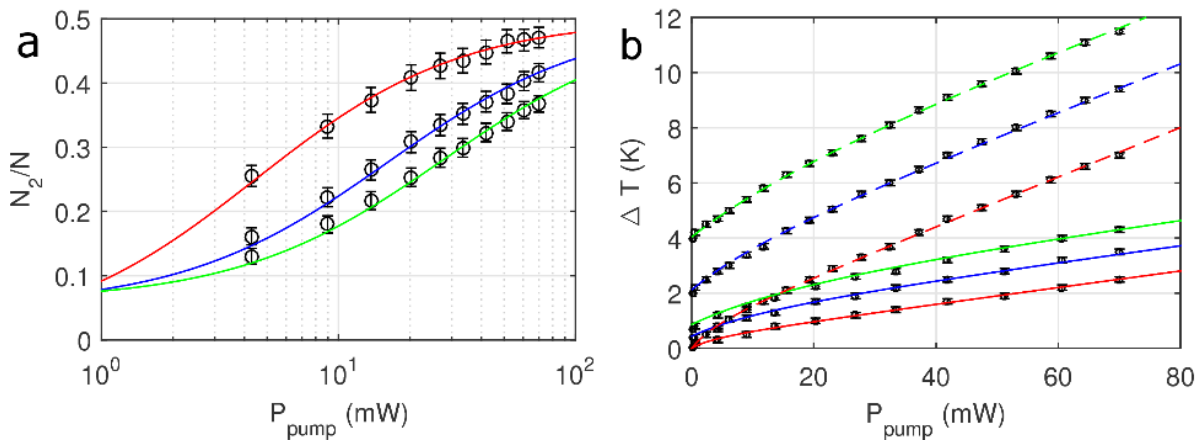

Fig. 1. (a) Dependence of inversion on pump light $(976 \mathrm{~nm})$ in a pristine fiber for different amount of signal light $-0 \mathrm{~mW}$ (red), $100 \mathrm{~mW}$ (blue) and $200 \mathrm{~mW}$ (green). (b) Temperature increase vs pump power in a pristine (full curve) and photodarkened (dashed). Same color scheme applies.

In parallel with the measurement of inversion, a temperature increase was monitored (Fig. 1(b)). Typical shape of curve with decreasing slope at low powers that becomes constant at higher pump powers revealed the two most important contributions to heating: linear absorption of pump and signal light and quantum defect. While the first (linear absorption) linearly grows with the power of pump light propagating in the fiber, the second (quantum defect) follows the inversion, which saturates at higher pump powers. The third mechanism, i.e. non-radiative decay, was also included due to the discrepancy at low pump powers, especially in the case of no signal light, where completely linear dependence of temperature rise on pump power was expected.

Two free parameters in the model, linear absorption coefficient at $976 \mathrm{~nm}$ and nonradiative decay rate were extracted from the fits and are shown in Fig. 2. Even in a pristine fiber there is a linear absorption at both pump and signal light, which cannot be attributed directly to PD process, but probably has its origin in other impurities in the optical fiber. After the fiber was photodarkened, linear absorption at $976 \mathrm{~nm}$ increased for a factor of 3, while at 
$1030 \mathrm{~nm}$ more than 6-fold increase was measured. On the other hand non-radiative decay rate $A_{\mathrm{NR}}$ was set to zero for a pristine fiber. Multi-phonon relaxation rate in ytterbium-doped silica glass is indeed negligible due to low phonon energy compared to energy spacing between laser levels of ytterbium ion. However, after modifications of fiber glass during PD process, new non-radiative decay channel opens, which can be observed as the shortening of fluorescence lifetime according to $1 / \tau=1 / \tau \mathrm{r}+1 / \tau \mathrm{nr}$, where $\tau, \tau \mathrm{r}=1 / A_{21}$ and $\tau_{\mathrm{NR}}=1 / A_{\mathrm{NR}}$ are fluorescence, radiative and a non-radiative lifetime, respectively. Measured fluorescence lifetimes of pristine and photo darkened fiber are $0.83 \pm 0.02 \mathrm{~ms}$ and $0.77 \pm 0.01 \mathrm{~ms}$ long, respectively, giving a non-radiative decay time of $11 \pm 5 \mathrm{~ms}$ or non-radiative decay rate of 94 $\pm 46 \mathrm{~s}^{-1}$. The latter can be also extracted from our heat source model with comparable uncertainty $\left(A_{\mathrm{NR}}=104 \pm 45 \mathrm{~s}^{-1}\right.$ - see Fig. 2).
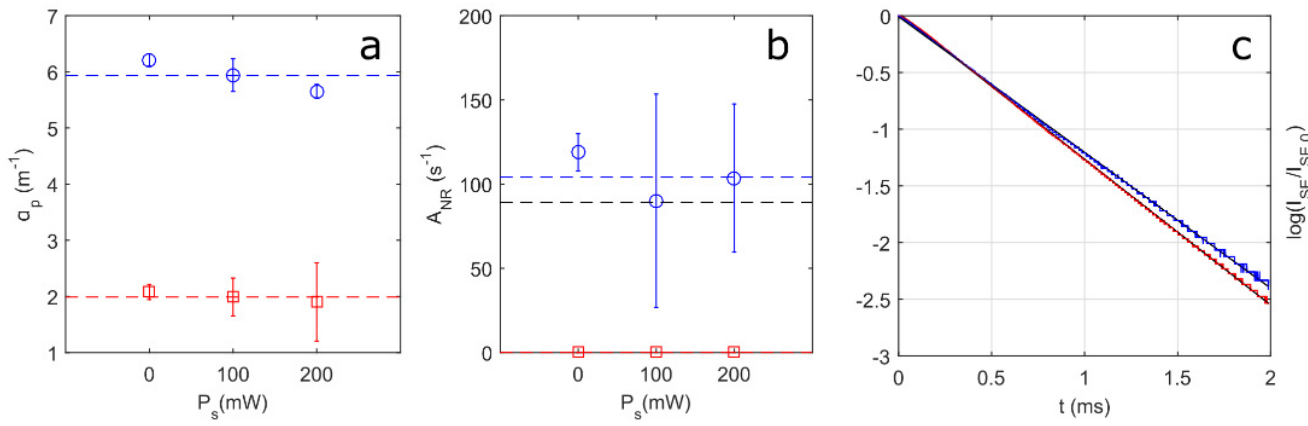

Fig. 2. (a,b) Fit results of model (6) for pristine (red) and photodarkened (blue) fiber and mean values (dashed curves). In (a) absorption coefficients (sum of intrinsic and PD induced loss) at pump wavelength is shown and in (b) non-radiative decay rates (set to 0 for pristine fiber). The black dashed horizontal line is non-radiative decay rate as extracted directly from fluorescensce lifetime measurement shown in (c) assuming a single-exponential decay. $I_{\mathrm{SE}}$ is a power of detected spontaneously emitted light emitted after the end an excitation pulse and $I_{\mathrm{SE}, 0}=I_{\mathrm{SE}}(t=0)$.

Finally, our heating model was tested on a core pumped fiber amplifier made from the same type of active fiber as in the model calibration (photodarkened prior to this test) with 4.5 $\mathrm{mW}$ of signal and $330 \mathrm{~mW}$ of pump light launched at the input. The inversion, pump and signal power along the fiber can be calculated with a standard fiber amplifier model solving for steady state. The obtained values are next used as input data for heating model to predict temperature elevation. In Fig. 3 measured values of inversion and temperature rise are compared to model results.

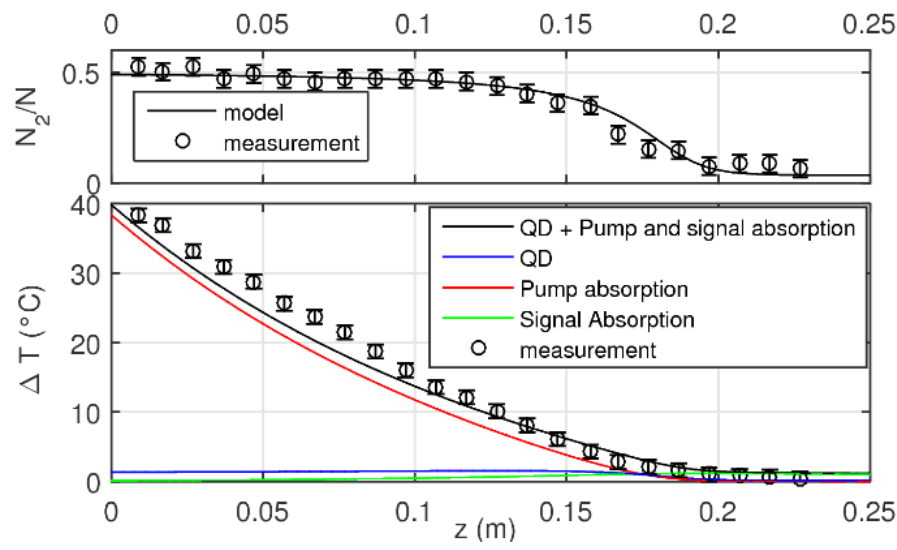

Fig. 3. Inversion (up) and temperature rise (below) along fiber preamplifier. 
As expected, inversion is saturated at the beginning of the amplifier, but then quickly falls down once the signal light prevails over pump. The measured and predicted position of this roll over (around $17 \mathrm{~cm}$ ) match very well. The temperature rise as well almost completely overlaps with the model predictions. Slightly higher measured temperature rise could be attributed to the fact, that model was calibrated on a $2 \mathrm{~mm}$ long piece of fiber, with not completely negligible heat transfer by conduction to passive fiber at both active fiber's ends. The dominant heating mechanism is identified to be the absorption of pump light being 4 times larger than absorption of signal light. The contribution of quantum-defect to temperature rise is almost 20 times weaker than the one of pump light absorption at the input of the fiber amplifier where temperature is the highest (more than $65^{\circ} \mathrm{C}$ ). Such high temperature increase at low pump powers should raise a concern, because for higher, yet realistic pump powers $(\sim 1 \mathrm{~W})$ temperature levels can be reached $\left(\sim 120^{\circ} \mathrm{C}\right)$ at which damage to fibers will be made if no precautions in cooling the system are made.

From our model we can now extrapolate the impact of PD in different fiber laser systems on heating for the active fiber characterized in our experiment. In Fig. 4, the ratio of PD- and quantum defect-related heating is shown in contour plot for different powers of signal and pump light. A linear dependence of equilibrium PD excess loss on inversion was assumed, as was measured in $[9,14]$. The PD-related heating dominates significantly at lower signal powers, where inversion is saturated and therefore the PD-induced excess loss is the highest. At the same time heating due to quantum defect is weak, because of lower rate of stimulated emission. The heat load in this region ( $<1 \mathrm{~W}$ of signal or pump power) is indeed not high $(<10$ $\mathrm{W} / \mathrm{m}$ ) and can be easily manageable with a simple cooling scheme. Yet, as quantum defect contribution is even several times smaller than real total heat load, any active cooling is often ignored, what can cause damage to the system after PD reaches a critical level. At much higher pump and seed powers $(\sim 1 \mathrm{~kW})$ the situation is different: quantum defect-related heating is already that high, that active cooling system is by default required. PD contribution is still comparable to quantum defect and the additional net heat load must be taken into account.

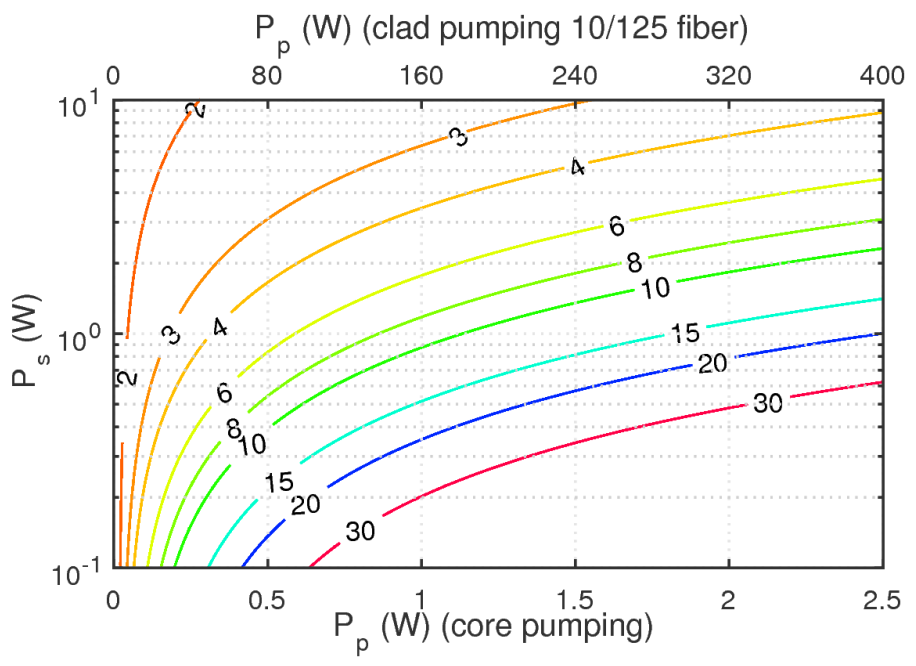

Fig. 4. Ratio of $\mathrm{PD}$ and $\mathrm{QD}$ heat source at different signal and pump powers.

\section{Conclusion}

In this paper we presented a heat-load model of a fiber laser, in which three main contributions due to intrinsic absorption, quantum defect and PD are included. A way to distinguish between quantum defect and PD contributions by monitoring temperature in time was presented. While main impact of PD in ytterbium doped fiber is additional absorption of 
both pump and signal light, additional non-radiative decay channel was measured which shortens the lifetime of upper laser level and was considered as additional heat source. It was found to have negligible impact on overall temperature rise. The very good agreement between the model and experimental result proves the capability of temperature monitoring (if possible together with fluorescence) to predict the distribution of pump and signal light within fiber laser or contrary. The presented model and test procedures can also help engineers in the design stage to select proper cooling scheme and importantly, to not underestimate the impact PD has on heating of fiber lasers and amplifiers.

\section{Funding}

Slovenian Research Agency (P2-0270, P2-0392) Projects L2-6780, L2-8183.

\section{Comment}

The article relates to SPS Operation entitled Building blocks, tools and systems for future factories - GOSTOP. 